\title{
Mini-review on the glucose metabolism modifications after pancreatic resection
}

\author{
TIMOFTE Daniel $^{1 *}$, IONESCU Lidia ${ }^{1}$, OCHIUZ Lacramioara ${ }^{1}$ \\ 1“Gr. T. Popa" University of Medicine and Pharmacy, lasi, Romania \\ *dantimofte@yahoo.com
}

Keywords: glucose, pancreatic cancer, diabetes.

\begin{abstract}
Pancreatic cancer is one of the most severe malignant disease, with an extreme degree of lethality, considering that the survival rate at 5 years is up to $4 \%$. In addition, a major disadvantage of this disease is the fact that the diagnosis is determined very late in the evolution of the disorder, despite the development of new technologies. In this way, the main symptoms are occurring later on, when the tumour is well to advanced and totally unresectable. Up to now surgery is the only modality that can provide a greater chance of survival, but unfortunately the pancreatic resection has many unknowns and controversies around it. Moreover, the studies on endocrine pancreatic function after resection are very few and somehow controversial. In this way, in the present minireview we will describe the most relevant experimental data regarding the post-resection pancreatogenic diabetes, the pancreatic polypeptide PP and the pancreatic glucose metabolism after resection or the glucose metabolism after partial or total pancreatectomy.
\end{abstract}

\section{Introduction}

Pancreatic cancer is one of the most severe malignant disease, with an extreme degree of lethality, considering that the survival rate at 5 years is up to $4 \%[1,2]$.

In addition, a major disadvantage of this disease is the fact that the diagnosis is determined very late in the evolution of the disorder, despite the development of new technologies. In this way, the main symptoms are occurring later on, when the tumour is already locally advanced and unresectable.

Up to now surgery is the only modality that can provide a greater chance of survival, but unfortunately the pancreatic resection has many unknowns and controversies around it $[1,2]$.

Moreover, the studies on endocrine pancreatic function after resection are very few and somehow controversial. This is probably due to long metabolic consequences after the cephalic duodenopancreatectomy (CPD) irrespective of the resection type, but rather dependent on the pancreas resection remnants and the indication for resection.

In fact, the postoperative consequences after different resections types and drainage procedures for pancreatic cancer are synthesised in Table 1.

Thus, a predictive assessment of the metabolism is difficult to be made, with limits of metabolic changes from mild to major. However, in general the postoperative changes are usually severe, but approximately $17-25 \%$ of patients will develop insulin-dependent diabetes.

Table 1. The postoperative consequences after different resections types and drainage procedures for pancreatic cancer

\begin{tabular}{|l|l|l|l|}
\hline Distal pancreatectomy & New diabetes & $21-40 \%$ & Morrow [3] \\
\hline $\begin{array}{l}\text { Duodenum preserving cephalic } \\
\text { pancreatectomy }\end{array}$ & New diabetes & $2-15 \%$ & Beger [4] \\
\hline $\begin{array}{l}\text { Pylorus preserving cephalic } \\
\text { duodenopancreatectomy }\end{array}$ & New diabetes & $12 \%$ & Traverso [5] \\
\hline CPD Whipple & New diabetes & $17 \%$ & Schwarz [6] \\
\hline Total pancreatectomy & Difficult diabetes & $100 \%$ & Andrén-Sandberg [7] \\
\hline
\end{tabular}




\section{Experimental data}

In this way, most of experimental data suggested that the glucose intolerance occurrence after CPD is not only due to insufficient insulin. Thus, Krusch et al. have shown that reducing beta cells after $80 \%$ proximal pancreatectomy in dogs causes low levels of insulin and impaired peripheral response to the glucose tolerance test [8].

Also, abnormalities were observed regarding fasting hyperglycaemia, high levels of glucose and a decrease in glucose metabolism (e.g. $\mathrm{k}$ value). Moreover, pancreatic resection with venous drainage shunted to the systemic circulation was associated with lasting high levels and $\mathrm{k}$ values, which remain low. These data are suggesting that surgical alterations of the pancreas consist of impaired glucose metabolism independent of the peripheral insulin levels.

In addition, patients suffering from pancreatogenic diabetes behave differently from those which are insulin-dependent. In this way, the patients that are experiencing some evident difficulties in controlling diabetes (e.g. the so-called "brittle diabetics") could become unexpectedly hypoglycaemic during insulin therapy, unrelated to food intake or exercise. In this way, Nosadini et al. studied the way insulin is acting in those pancreatogenic diabetes patients, as compared to type I diabetics to evaluate insulin sensitivity [9]. Thus, they reported an increased sensitivity of the extrahepatic tissues in physiological hyperinsulinemia and insulin in increased amounts bound to the erythrocytes receptors in patients with pancreatogenic diabetes. Moreover, the increased binding of the insulin is due to the peripheral sites increase which bound the insulin rather than to a change in receptor affinity. Even more, the destruction of insulin was analysed after the discontinuous infusion of insulin, while the insulin plasma clearance was significantly higher in patients with pancreatogenic diabetes, as compared to those with type I diabetes. Thus, there is an increase in the peripheral insulin receptors in response to an insulin deficit, making these patients sensitive to hormone replacement [9].

We can affirm in this way, based on the aforementioned aspects that long-term metabolic consequences after the CPD differ even after the same type of operation due to the different characteristics of the pancreas resection. In general, the postoperative changes are moderate to severe, but $17 \%-25 \%$ of patients will develop insulin-dependent diabetes. The experimental results are also suggesting that insulin-independent glucose metabolism after the pancreatic resection is created for a large proportion of absorbed glucose. This may explain why patients with pancreatogenic diabetes behave differently than other diabetics sensitive to insulin therapy. These patients that are experiencing some evident difficulties in controlling diabetes can become unexpectedly hypoglycaemic during therapy with insulin, unrelated to food intake or exercise. Thus, it was observed an increased sensitivity of the extrahepatic tissues in physiological hyperinsulinemia and insulin in increased amounts bound to the erythrocytes receptors in patients with pancreatogenic diabetes. In this way, there is an increase in the peripheral insulin receptors in response to an insulin deficit, making these patients sensitive to hormone replacement.

\section{Post-resection pancreatogenic diabetes}

The actions of insulin include a wide range of effects on a wide variety of tissues. Insulin increases the transport of ions, glucose and amino acids across the cell membrane, has anabolic effects as protein synthesis and lipogenesis and it also has secondary growth promoting effects to stimulate the synthesis of DNA and RNA.

It has to be mentioned that the diabetes resulted after the pancreatic resection differs from Type I diabetes (juvenile or insulin-dependent) and the Type II (adult or non-insulin-dependent) in several ways. Unlike Type I diabetes, patients with pancreatogenic diabetes rarely develop ketoacidosis, and the degree of hyperglycaemia is reduced in the majority of the situations. Moreover, when compared to type II diabetes, which has as the main characteristic the deep resistance to insulin, the patients undergoing major pancreatic resections are really sensitive to insulin administration.

Also, these patients have an increased serum level of gluconeogenic precursors such as lactate and alanine [10], while distinct clinical and metabolic aspects were assigned to pancreatic glucon 
secretion deficiency [11]. Also, during fasting, glucagon is responsible for maintaining proper glucose production at hepatocellular level by stimulating glycogenolysis and gluconeogenesis [12]. Pancreatogen diabetes has also elevated levels of serum insulin and has a minimum or no response to food intake, in contrast to the type I diabetic insulin serum, which is normal or elevated and who has an exaggerated response to the ingestion of carbohydrates [13].

In addition, the liver plays a central role in the regulation of glucose metabolism. The liver capacity to produce glucose in conditions of stress or prolonged fast and retrieve glucose after the ingestion of carbohydrates is essential for carbohydrate metabolism. These functions are closely dependent on three hormones secreted by the pancreas: insulin, glucagon and pancreatic polypeptide.

In this way, the insulin-secreting beta cells in the pancreas are homogeneously distributed, while the glucagon secreting alpha cells and PP cells which secrete pancreatic polypeptide are mainly distributed in the tail, respectively the head of the pancreas. Also, the hepatic insulin resistance and uncontrolled production of glucose are the boundary of the pancreatogenic diabetes and are dependent on the relative insulin deficiency, glucagon and PP [14].

In addition, for all patients undergoing total pancreatectomy, about 25-40 units of insulin per day are sufficient for blood sugar control. This usually used recommendation increases in the recovery months most commonly due to the improved appetite and carbohydrate intake [13].

All these aspects are generally synthesized in the Table 2.

Table 2. A schematic comparison between postresection pancreatogen diabetes, as compared to classical forms.

\begin{tabular}{|l|l|l|l|}
\hline Variables & Type I diabetes & Type II diabetes & $\begin{array}{l}\text { Pancreatogenic diabetes } \\
\text { "type III" }\end{array}$ \\
\hline ketoacidosis & frequent & rare & rare \\
\hline hyperglycaemia & severe & usually mild & mild \\
\hline hypoglycaemia & frequent & rare & frequent \\
\hline $\begin{array}{l}\text { peripheral insulin } \\
\text { sensitivity }\end{array}$ & Normal/increased & decreased & increased \\
\hline $\begin{array}{l}\text { hepatic insulin } \\
\text { sensitivity }\end{array}$ & normal & increased & decreased \\
\hline Insulin levels & decreased & increased & decreased \\
\hline glucagon levels & normal/increased & normal/increased & decreased \\
\hline PP level & increased & increased & decreased \\
\hline typical age of onset & Child/teenager & adult & Any age \\
\hline
\end{tabular}

Moreover, as emphasized by Frey et al. [15], the incidence of postoperative diabetes is directly related to the extension of pancreatic tissue resection. In this way, patients who require total or almost total pancreatectomy pay a significant price in altered glucoregulatory hormone secretion. These patients that require distal pancreatectomy or only a limited excision of the cephalic pancreas are developing diabetes far less frequently than those that require CPD or total or almost total pancreatectomy.

Also, patients with CPD have a good metabolic status and, although a significant number of them require insulin replacement therapy, many patients manage to control blood sugar with oral antidiabetics. Moreover, antrectomy related complications are avoided by preserving the pylorus, while the postoperative endocrine dysfunction is comparable at both processes of 
duodenopancreatectomy. Also, the cephalic pancreatectomy with the preservation of the duodenum seems to offer protection in the postoperatively diabetes, as compared to CPD. However, it is important to note that these patients already have at the time of surgery alteration of the whole pancreatic tissue or have a high risk for any reduction in the active pancreatic endocrine tissue. Thus, these aspects may influence the incidence of diabetes after surgery depending on the different types of resection.

\section{The pancreatic polypeptide PP and the pancreatic glucose metabolism after resection}

In this way, despite an increase in peripheral insulin receptor availability, the pancreatogen diabetes comes with a decrease in the hepatic insulin receptor sensitivity [16]. This paradoxal effect is probably due to a competitor deficit of PP, which makes the liver resistant to the suppressor effects of insulin for hepatic production of glucose [17]. Moreover, the result of the increase in hepatic responsiveness to glucagon and the decrease of hepatic sensitivity is a growth in endogenous glucose production, which causes hyperglycaemia with a high hypoglycaemic result in regards to the exogenous insulin for the patients with pancreatogen diabetes.

Also, the PP pancreatic polypeptide is released in a biphasic manner in response to food ingestion and inhibits exocrine pancreatic secretion and gallbladder contraction. Moreover, PP binds to areas of the brainstem, and interpeduncular nuclei adjacent bind to vagal or cholinergic nuclei, while the PP receptors have also been identified in rat hepatocyte membranes.

Considering the importance of the animal models in the present research status [18-20], studies on canine models of chronic pancreatitis and PP deficiency also demonstrated hepatic resistance to insulin with inadequate production of glucose in the liver, despite normal levels of insulin. Moreover, PP deficient status occurs frequently after surgical resection, considering that the hormone is predominantly localized in the cephalic pancreas. Also, in order to further define the role of PP and to demonstrate whether surgically induced deficiency of PP is associated with abnormal glucose metabolism, Seymour paper described male patients which had pancreatic resections for pancreatic traumas (bruises or wounds) [21]. In this way, the resected patients deficient in PP response had severe dysfunctions in liver insulin response. Moreover, following the PP infusion PP-deficient group was indistinguishable from control subjects in terms of suppression of hepatic glucose production. Also, completely reversible hepatic insulin resistance after administration of PP was again cleared one month later.

These studies provide clear information about the role of PP in hepatic insulin sensitivity, while subsequent studies suggested that hepatic insulin receptors continuing validity is dependent on the PP. Moreover, it has been shown on rats with chronic pancreatitis that PP deficiency is associated with decreased insulin sensitivity in the hepatic receptor, which is also a reversible process after the administration of PP. Even more, it was later demonstrated that the PP regulates the expression of hepatic insulin receptor gene in healthy rats and in those with decreased sensitivity of insulin receptors in the pancreatogenic diabetes due to chronic pancreatitis. In this way, PP deficit is a potentially reversible pathophysiological factor contributing to impaired glucose metabolism after cephalic or total pancreatectomy, as a result of the decreased sensitivity in the hepatic insulin receptor and the gene expression of these receptors.

\section{Glucose metabolism after partial pancreatectomy}

Regarding the glucose metabolism after partial pancreatectomy, the group of Buchler et al. [22] compared the endocrine function after PCD-PP after cephalic pancreatectomy with preservation of the duodenum (CP-PD) for chronic pancreatitis. Preoperatively, blood glucose, insulin and glucagon were similar in the 2 groups in the fasting period and after lunch. Also, at 6 months after surgery patients in the PCD-PP group showed glucose intolerance after a standard meal (median glucose $130 \mathrm{mg} / \mathrm{dl}$ after $150 \mathrm{~min}$ ) while glucose tolerance did not changed in the CP-PD group (median glucose $88 \mathrm{mg} / \mathrm{dl}$ ). Even more, preoperative insulin secretion was similar in the 2 groups. After resection, the ability of insulin secretion in the group of PCD-PP decreased from 29.4 to 24.5 
microU / $\mathrm{ml} / \mathrm{min}$, and CP-PD group increased from 29.4 to 32.1. We also have to mention that compared to the preoperative levels, the values of serum glucagon and glucagon secretion capacity have decreased in the two groups. This could suggest that preservation of the duodenum (or of the pancreatic tissue from the ventral pancreas which contains insulin and PP) is responsible for the postoperative improvement in glucose tolerance.

In addition, there are other reported studies about the beneficial effect of duodenum preservation on glucose homeostasis [23]. In this way, a comparison between several processes such as Whipple, Beger and Frey [24] revealed that although the incidence of new diabetes or a worsening preexisting one was $22 \%$ in the group of 11 patients who received the Whipple procedure, none of the 9 patients which received the Beger procedure showed any exacerbation of the diabetes. Moreover, one patient became insulin- dependent after the Beger procedure. Even more, none of the patients operated with the Frey procedure showed any exacerbation of the diabetes. Thus, it seems that these findings are supporting the concept that the preservation of the duodenum or a portion of the ventral pancreas maintains glucose tolerance and avoids the appearance of new diabetes.

In conclusion, there are evidences that PC-PD is less aggressive than other hemipancreatectomies in regards to the changes of the glucose metabolism. In this way, as previously mentioned, the observations of some studies suggested that the preservation of the duodenum (or ventral pancreatic tissue that secretes insulin and PP) is responsible for improving postoperative glucose tolerance

\section{Glucose metabolism after total pancreatectomy}

It is very well known that the hormones from the digestive tract are determining both stimulation and inhibition on the exocrine and endocrine pancreatic secretions. The effect of total pancreatectomy, in regards to the source of digestive hormones extinction was compared in 8 patients, after total pancreatectomy with other healthy subjects [25]. In this way, the aforementioned study demonstrated that patients with pancreatectomy had a total deficit of endogenous insulin, glucagon and PP. Moreover, it seems that there is a deficient release of gastrin after lunch stimulation due to the effect of antrectomy, while the levels of other hormones such as motilin, gastric inhibitory polypeptide and gastric inhibitory polypeptide (GIP) were not different from healthy subjects.

Also, the enteroglucagon, which is believed to slow down intestinal transit and stimulate the growth of gastrointestinal mucosa net, increases in resected patients after stimulation through lunch. The explanation for this fact may lie in the increasing transit time through the stomach after gastrojejunal anastomosis or an increased expression of the preproglucagon in the digestive tract.

Moreover, the GIP and glucagon-like peptide-1 (e.g. an intestinal cleavage product of the preproglucagon) are acting as a stimulant intestinal hormone of insulin release.

Moreover, changes in the secretion of these hormones may occur as a compensatory mechanism after the operative loss of pancreatic tissue. Thus, total pancreatectomy may determine not only a deficiency of pancreatic hormones, but also dysfunctions of other digestive hormones which are important for the general metabolism.

Total pancreatectomy also determines a glucagon deficiency. In this way, it has been found in ducks that after the TP, glucagon levels is decreased to $45 \%$ of the preoperative initial level, immediately after surgery [10]. Also, within 80 minutes, the plasma is removing $94 \%$ of the initial glucagon-like immunoreactivity, while positive highly significant correlation was found between the level of plasma glucagon and glucose levels.

Even more, in certain animals hypoglycaemic seizures could occur in 2 hours after the end of surgery. Also, in humans glucagon deficiency in chronic pancreatitis was the primary cause for the reduction of hepatic glucose production in patients with PT and directly determines an increase in serum levels of gluconeogenesis precursors. In fact, correcting the glucagon deficit determines the increase of glucose turn-over, as well as a decrease in blood precursors for gluconeogenesis such as alanine, lactate, and pyruvate. 
Controversies still exist on the assumption that total pancreatectomy implies removing of all the sources which are producing glucagon in humans. Many animals such as rats, dogs and cats have enteric glucagon sources, and TP in these animals does not suppress the circulating level of glucagon. Also, several studies have investigated whether patients are deficient in glucagon after PT.

In this way, Barnes and Bloom have reported that in humans, after PT, there is no detectable immunoreactive circulating glucagon in the plasma. Other researchers have additionally detected varying amounts of glucagon in patients with TP. In one of these studies, they found biologically active glucagon in gel filtered plasma from resected patients [26]. The mean concentration of immunoreactive glucagon was significantly lower in resected patients, as compared to the healthy controls (18 vs. $49 \mathrm{pg} / \mathrm{ml}$ ) and the baseline in glucagon levels varied within a wide range (from 10 to $38 \mathrm{pg} / \mathrm{ml}$ ). Also, plasma glucagon was not increased by the amino acid arginine, suggesting that immunoreactive glucagon differs from glucagon from subjects with intact pancreas. While a source of enteral production of glucagon in humans may exist after TP, there is a major deficit in glucagon which in combination with insulin deficiency can result in a metabolic crisis [13].

As in the case of insulin, glucagon sensitivity is increased after TP. In fact, it has been found that the metabolic response to glucagon was significantly more pronounced in patients with TP, as compared to patients with type I diabetes. Thus, a chronic deficit of glucagon can change the effect that glucagon exerts on the liver, perhaps through an increase in the sensitivity of the glucagon receptor which has the effect of amplifying the hyperglycemic response.

Moreover, after glucose loading, glucagon serum was significantly increased in patients operated with the Whipple procedure, Billroth II reconstruction type and patients with Child procedure (pancreaticojejunostomy, choledochojejunostomy and gastrojejunostomy), suggesting that these factors were affected by the type of reconstruction performed on the digestive tract. Also the immunoreactive serum glucagon levels, including the pancreatic one, were slightly elevated on patients operated with Child procedure of distal pancreatectomy after the administration of glucose, suggesting that these paradoxical responses may be associated with the type of specific digestive reconstruction used

\section{Conclusions}

Up to now surgery is the only modality that can provide a greater chance of survival in the pancreatic cancer, but unfortunately the pancreatic resection has many unknowns and controversies around it. Moreover, the studies on endocrine pancreatic function after resection are very few and somehow controversial. In this way, in the present mini-review we described the most relevant experimental data regarding the post-resection pancreatogenic diabetes, the pancreatic polypeptide PP and the pancreatic glucose metabolism after resection or the glucose metabolism after partial or total pancreatectomy.

\section{References}

[1] V. Surlin, V. Bintintan, F. Petrariu, R. Dobrin, R. Lefter, A. Ciobică, D. Timofte, Prognostic factors in resectable pancreatic cancer, Rev Med Chir Soc Med Nat Iasi. 118 (2014) 924-31.

[2] D. Timofte, R. Danila, A. Ciobica, C. Diaconu, R. Livadaru, L. Ionescu, The relevance of some tumoral markers in patients with pancreatic cancer, Analele Stiintifice ale Universitatii" A1. I. Cuza" Din Iasi. 15 (2014) 51-58.

[3] C. Morrow, Chronic pancreatitis: long-term surgical results of pancreatic duct drainage, pancreatic resection, and near-total pancreatectomy and islet autotransplantation, Surgery. 96 (1984) 608-16.

[4] H. Beger, M. Buchler, Duodenum-preserving resection of the head of the pancreas in chronic pancreatitis with inflammatory mass in the head, World J Surg. 14 (1990) 83-7. 
[5] L. Traverso, R.A. Kozarek, Pancreatoduodenectomy for chronic pancreatitis: anatomic selection criteria and subsequent long-term outcome analysis, Ann Surg. 226 (1997) 429-35.

[6] R. Schwarz, D. Smith, Extent of lymph node retrieval and pancreatic cancer survival: information from a large US population database, Ann Surg Oncol. 13 (2006) 1189-1200.

[7] A. Andren-Sandberg I. Ihse, Factors influencing survival after total pancreatectomy in patients with pancreatic cancer, Ann Surg. 198 (1983) 605-10.

[8] D. Krusch, T. Pruett, G. Cornett, J. Hanks, Surgical alterations of the pancreas and insulinindependent glucose disposal, J Surg Res. 52 (1992) 476-82.

[9] R. Nosadini, Insulin sensitivity, binding, and kinetics in pancreatogenic and type I diabetes. Diabetes. 31 (1982) 346-55.

[10] H. Karmann, F. Laurent, P. Mialhe, Pancreatic hormones disappearance after total pancreatectomy in the duck: correlation between plasma glucagon and glucose. Horm Metab Res. 19 (1987) 538-41.

[11] R. Unger, Glucagon physiology and pathophysiology, N Engl J Med. 285 (1971) 443-9.

[12] S. Kreutzenberg, Glucose turnover and recycling in diabetes secondary to total pancreatectomy: effect of glucagon infusion, J Clin Endocrinol Metab. 70 (1990) 1023-9.

[13] L. Slezak, D. Andersen, Pancreatic resection: effects on glucose metabolism, World J Surg. 25 (2001) 452-60.

[14]. A. Fowden, W. Hay, The effects of pancreatectomy on the rates of glucose utilization, oxidation and production in the sheep fetus, Q J Exp Physiol. 73(1988) 973-84.

[15] C. Frey C. Child, W. Fry, Pancreatectomy for chronic pancreatitis, Ann Surg. 184 (1976) 40313.

[16] N. Seymour, Alterations in hepatocyte insulin binding in chronic pancreatitis: effects of pancreatic polypeptide, Am J Surg. 169 (1995) 105-9.

[17] D. Andersen, C Ruiz, C Burant, Insulin regulation of hepatic glucose transporter protein is impaired in chronic pancreatitis, Ann Surg. 219 (1994) 679-86.

[18]. A. Ciobica, V. Bild, L. Hritcu, M. Padurariu, W. Bild, Effects of angiotensin II receptor antagonists on anxiety and some oxidative stress markers in rat, Central European Journal of Medicine. 6 (2011) 331-340.

[19]W Bild, A Ciobica, Angiotensin-(1-7) central administration induces anxiolytic-like effects in elevated plus maze and decreased oxidative stress in the amygdala, Journal of affective disorders. 145 (2013) 165-171.

[20]. W. Bild, L. Hritcu, C. Stefanescu, A. Ciobica, Inhibition of central angiotensin II enhances memory function and reduces oxidative stress status in rat hippocampus, Progress in NeuroPsychopharmacology and Biological Psychiatry. 43 (2013) 79-88.

[21] N. Seymour, Reversal of abnormal glucose production after pancreatic resection by pancreatic polypeptide administration in man, Surgery. 104 (1988) 119-29.

[22] M. Buchler, Randomized trial of duodenum-preserving pancreatic head resection versus pylorus-preserving Whipple in chronic pancreatitis, Am J Surg. 169 (1995) 65-9.

[23] G. Aspelund, Improved outcomes for benign disease with limited pancreatic head resection, J Gastrointest Surg. 9 (2005) 400-9.

[24] H. Ho, C Frey, The Frey procedure: local resection of pancreatic head combined with lateral pancreaticojejunostomy, Arch Surg. 136 (2001) 1353-8.

[25] H. Dammann, Gut-hormone profile in totally pancreatectomised patients, Gut. 22(1981) 103-7.

[26] G. Boden, Glucagon deficiency and hyperaminoacidemia after total pancreatectomy, J Clin Invest. 65 (1980) 706-16. 